\title{
COMPUTER SIMULATION OF THE PESTICIDE DEPOSITION DISTRIBUTION IN HORIZONTAL DIRECTION SPRAY
}

\author{
Wanzhang Wang ${ }^{1}$, Tiansheng Hong ${ }^{2}$, Wenyi Liu ${ }^{1}$, Xiangfu $\mathrm{Li}^{1}$ \\ ${ }^{1}$ College of Mechanical and Electrical Engineering, Henan Agricultural University, 95 \\ Wenhua Road, Zhengzhou Henan Province 450002, P. R. China, Tel: +86-371-63558289, \\ Fax:+86-371-63558040,Email: wanzwang@gmail.com \\ ${ }^{2}$ College of Engineering, South China Agricultural University Guangzhou, China, 510642
}

Abstract: The objective of this study is taken to realize pesticide precision spray of fruit trees and the other crops and reduce the deposition losses outside the canopy when the real time sensing technology was used in the pesticide target spray. In this paper the Pesticide solution deposition distribution experiments were conducted with two different volume median diameter (VMD) hollow cone nozzles fixed in horizontal direction, to investigate the influence of spray pressure and spray ground speed on the spray deposition region. The probability distribution model of the pesticide deposition was constructed based on the experiments, and the pesticide spray distribution range was simulated by using Matlab statistic toolbox. The simulation result showed that the spray pressure and the ground speed had the great influence on the maximum spray distance. With the increase of the spray speed, the spray deposition distribution range decreases gradually, when the nozzle 200 is under the speed above $1.20 \mathrm{~km} / \mathrm{h}$ and nozzle 300 is under the speed above $2.22 \mathrm{~km} / \mathrm{h}$, the deposition range was reduced greatly. So the computer simulations make a reference for the choice of the spray control parameters.

Keywords: computer simulation, pesticide deposition, lognormal distribution, target spray

\section{INTRODUCTION}

It's essential that the spray solution distribution range of the level placed nozzle must be given for precision spraying. In order to reduce the pesticide

Wang, W., Hong, T., Liu, W. and Li, X., 2008, in IFIP International Federation for Information Processing, Volume 258; Computer and Computing Technologies in Agriculture, Vol. 1; Daoliang Li; (Boston: Springer), pp. 425-432. 
loss in target sprays, the level spray distribution should be controlled by spray pressure and spray ground speed. It is known that level spray range of the droplets has relationship with quality of the droplet, nozzle moving velocity and the air conditions. The flying of the droplets has turbulence flow state, mix flow state and laminar flow state. Flying distance of single droplet can be calculated on the given conditions (Wanzhang Wang, 2005). Phase Doppler particle analysis under different pressure shows that with the increase of the pressure, the speed of big spray droplet increases rapidly and small droplet speed increases slowly. The higher the pressure of pesticide spray droplet, the greater the distinction of speed becomes in the spray droplet spectrum (Giles et al., 1992). The spray ground speed affects the spray solution distribution not only in the direction of the spray, but also in the moving direction. Horizontal movement of the nozzle is equivalent to the airflow moving relative to the nozzle act on the spray droplet, this will change the movement direction of the droplet, thereby the spray scope is reduced and the spray solution distribution is changed (China Agricultural University. 1999). When the speed of the nozzle is low, it only has effect on the movement of small droplet. When the speed of the nozzle is big, it has big effect on the whole spray cloud. But in actual spray, the size and speed of the spray droplet out of the nozzle are different. It is difficult to quantify the scope of the droplet sediment because of the influence of the spray velocity and many other complex factors. This thesis uses the method of pesticide spray experiment to obtain the changing regularities about the deposition distribution by the change of spray ground speed and pressure, and then carry through the computer simulation analysis.

\section{PESTICIDE SOLUTION DISTRIBUTION}

\subsection{Pesticide spray solution distribution test}

Two hydraulic hollow cone nozzles are used in experiments. Their models are type 320 and type 200 produced in Ikeuchi, Japan. Their VMD are $210 \mu \mathrm{m}$ and $130 \mu \mathrm{m}$ respectively. The diffuser angle of two nozzles is $80^{\circ}$ and operating pressure is from $0-7 \mathrm{MPa}$. Experiments of different spray pressures and spray ground speed have been designed on the spray test stand. The test stand is made of two or more $960 \times 2000 \mathrm{~mm}$ tables, each has 12 "V" shaped groove and the centerline distance of the "V" shaped groove is $80 \mathrm{~mm}$. 
Figure 1 shows the pesticide spray solution distribution histogram of the experiments in different pressure and speed with two type nozzles. It can be seen from the pesticide spray distribution histogram that, the pesticide spray distribution shows skew distribution in the spray pressure and spray speed experiments. In order to study the rule of the pesticide spray solution distribution, three parameters lognormal distribution functions are chosen to construct the probability distribution model of it. The three parameters lognormal distribution probability density function is shown below.

$$
f[\ln (x-d)]=\frac{1}{\sqrt{2 \pi} \sigma} \exp \left[-\frac{[\ln (x-d)-\mu]^{2}}{2 \sigma^{2}}\right]
$$

Where $d$ is position parameter, which represents the minimum distance of the pesticide deposition; $\sigma$ is the standard deviation, which shows the centralize degree of the pesticide spray, when $\sigma$ is small the spray sediment scope becomes narrow, and $\sigma$ big the scope becomes wide; $\chi$ is random variable which represents the spray range.

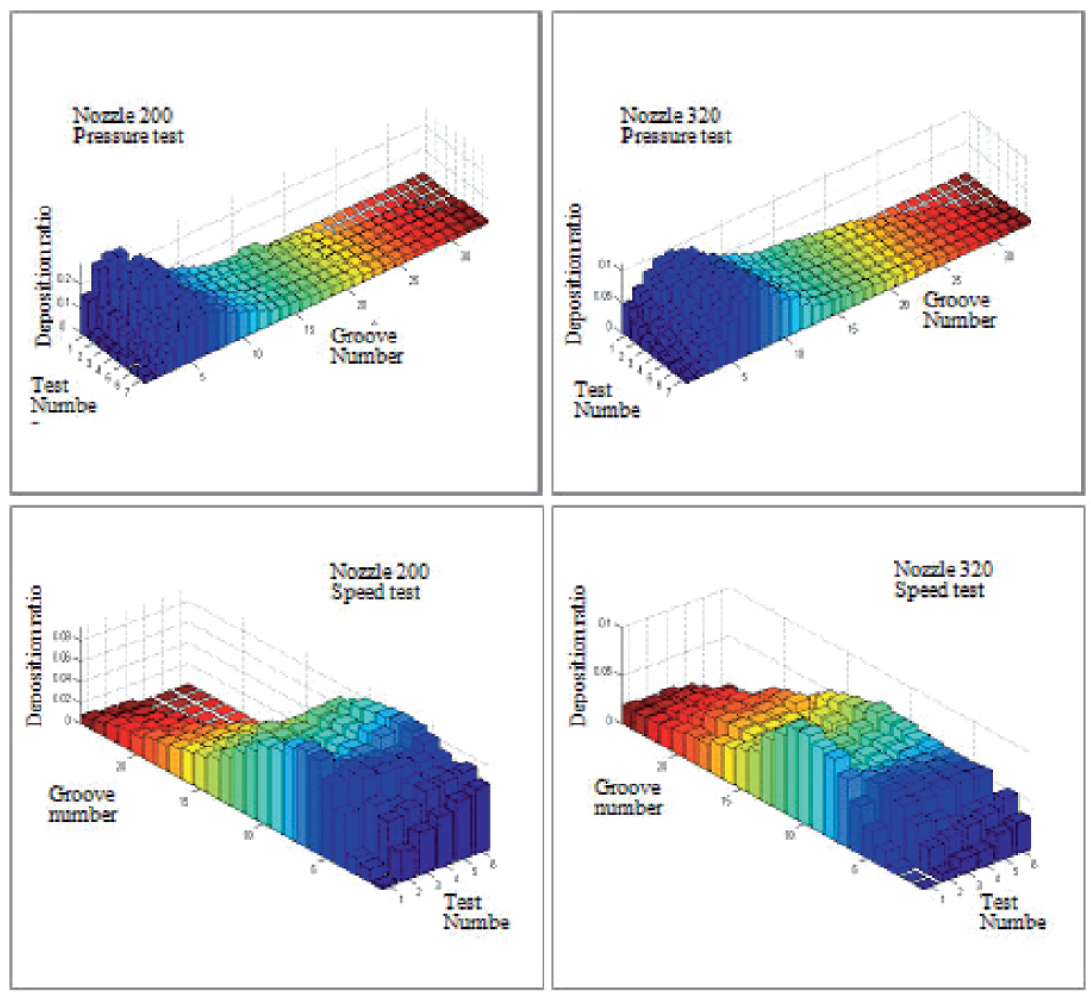

Figure 1. Distribution histogram of pesticide spray deposition 


\subsection{Lognormal distribution model}

The method of quick optimization parameter estimation of three parameters lognormal distribution function is chosen to calculate $d$ according to the test datum (Liu Dianrui et al., 1995).

$$
d=\frac{x_{\text {mo }}^{2}-x_{\text {min }} x_{\text {max }}}{2 x_{m o}-x_{\min } x_{\max }}
$$

Where $\mathrm{x}_{\operatorname{mo}}, \mathrm{x}_{\min }, \mathrm{x}_{\max }$ are respectively the mode, minimum and maximum of the test sample.

And $2 \mathrm{x}_{\operatorname{mo}}-\mathrm{x}_{\min } \mathrm{x}_{\max } \neq 0$

Standardize formula (1) and Let standard normal distribution value as:

$$
D=\frac{\ln (x-d)-\mu}{\sigma}
$$

Then $\ln (x-d)=\mu+\sigma D$

Formula (1) corresponding to the cumulative probability distribution function can be written in standard normal form:

$$
F[\ln (x-d)]=\frac{1}{\sqrt{2 \Pi}} \int_{0}^{\ln (x-d)} \exp \left(-\frac{D^{2}}{2}\right) d D
$$

It can be calculated according to the experimental data:

$$
F\left[\ln \left(x_{i}-d\right)\right]=\sum_{i=1}^{i} v_{i} / \sum_{i=1}^{n} v_{i}
$$

Where $i$ is spray test groove number; $x_{i}$ is the distance from the nozzle to the number $i$ groove centerline; $v_{i}$ is the spray solution collection volume of the number $i$ groove.

$D_{i}$ can be calculated by the inverse function of formula (3), then a set of data $\left(\ln \left(x_{i}-d\right), D_{i}\right)(i=1,2,3 \ldots, n)$ can be obtained. The parameters $\mu, \sigma$ and correlation coefficient $R$ can be calculated by linear regression analysis. When the absolute value of the correlation coefficient is greater, the linearity of the function is better, and the consistency of the lognormal distribution function is better. Then the normal probability distribution model of the pesticide spray solution distribution can be established:

$$
F(x)=\frac{1}{\sqrt{2 \Pi} \sigma x} \int_{0}^{x} \exp \left(-\frac{[\ln (x-d)-\mu]^{2}}{2 \sigma^{2}}\right) d x
$$

The minimum distance $\mathrm{d}$ change very little under the different spray ground speed in the same spray pressure and under different spray pressure in same spray ground speed. In order to simplify the calculation according to the experiment datum, $\mathrm{d}$ is regarded as constant in pressure experiment and speed experiment. Table 1 and 2 shows respectively the fitting results of pressure and speed experiment for the nozzle 200. Table 3 and 4 shows 
respectively the fitting results of pressure and speed experiment for the nozzle 320 .

Table 1. Nozzle 200 distribution parameters of pressure test $\mathrm{d}=584$

\begin{tabular}{llllllll}
\hline $\mathrm{P} / \mathrm{MPa}$ & 0.75 & 0.98 & 1.18 & 1.41 & 1.66 & 1.94 & 2.23 \\
\hline $\mathrm{R}^{2}$ & 0.959 & 0.969 & 0.972 & 0.955 & 0.968 & 0.983 & 0.964 \\
$\sigma$ & 0.771 & 0.715 & 0.727 & 0.723 & 0.770 & 0.694 & 0.769 \\
$\mu$ & 5.716 & 6.077 & 6.122 & 6.239 & 6.318 & 6.546 & 6.483 \\
\hline
\end{tabular}

Table 2. Nozzle 200 distribution parameters of ground speed test $\mathrm{d}=0$

\begin{tabular}{lllllll}
\hline $\mathrm{V} / \mathrm{km} / \mathrm{h}$ & 0.51 & 1.21 & 1.91 & 2.60 & 3.30 & 4.00 \\
\hline $\mathrm{R}^{2}$ & 0.99 & 0.969 & 0.959 & 0.915 & 0.914 & 0.897 \\
$\sigma$ & 0.437 & 0.501 & 0.497 & 0.432 & 0.432 & 0.399 \\
$\mu$ & 6.747 & 6.583 & 6.564 & 6.497 & 6.447 & 6.393 \\
\hline
\end{tabular}

Table 3. Nozzle 320 distribution parameters of pressure test $\mathrm{d}=442$

\begin{tabular}{llllllll}
\hline $\mathrm{P} / \mathrm{MPa}$ & 0.67 & 0.87 & 1.06 & 1.29 & 1.53 & 1.78 & 2.06 \\
\hline $\mathrm{R}^{2}$ & 0.954 & 0.960 & 0.971 & 0.974 & 0.973 & 0.978 & 0.979 \\
$\sigma$ & 0.652 & 0.654 & 0.657 & 0.639 & 0.615 & 0.635 & 0.625 \\
$\mu$ & 6.222 & 6.301 & 6.420 & 6.487 & 6.536 & 6.578 & 6.647 \\
\hline
\end{tabular}

Table 4. Nozzle 320 distribution parameters of ground speed test $\mathrm{d}=0$

\begin{tabular}{lllllll}
\hline $\mathrm{V} / \mathrm{km} / \mathrm{h}$ & 0.51 & 1.21 & 1.91 & 2.60 & 3.30 & 4.00 \\
\hline $\mathrm{R}^{2}$ & 0.996 & 0.965 & 0.958 & 0.951 & 0.905 & 0.907 \\
$\sigma$ & 0.379 & 0.533 & 0.559 & 0.555 & 0.467 & 0.458 \\
$\mu$ & 6.906 & 6.780 & 6.775 & 6.767 & 6.678 & 6.616 \\
\hline
\end{tabular}

\section{COMPUTER SIMULATION OF THE PESTICIDE DISTRIBUTION}

Establishing $\mathrm{X}$-axis in the direction of nozzle spraying, and $\mathrm{Y}$-axis in the direction of nozzle moving, the deposition of the spray droplet on the $\mathrm{X}$ and $\mathrm{Y}$ direction can be viewed as two independent probability event, and the probability density function $\mathrm{f}(\mathrm{x}, \mathrm{y})=\mathrm{f}(\mathrm{x}) \bullet \mathrm{f}(\mathrm{y})$ can be constructed according to test datum of the horizontal fixed nozzle above the plane area. 
The test datum under different spray pressure and speed shows that the pesticide spray solution distribution has normal distribution on the Y-axis. By construct two-dimensional probability density function of the pesticide spray distribution together with the normal distribution function on the $\mathrm{Y}$-axis and lognormal distribution function on the $\mathrm{X}$-axis, the simulation program can be compiled using Matlab6.0 statistic toolbox function Logndf and Normdf (Su Jin et al., 2000). Spray solution deposition distribution on the plane area below the nozzle can be computed by the Monte Carlo simulation with a given reasonable boundaries. A visual representation is obtained by the surface and contour diagram using Matlab drawing function to know where and how much the pesticide deposited. And then get the law of spray distribution change with the spray pressure and speed. Figure 2 shows the pesticide distribution of the static nozzle 200 under pressure 1.2 MPa. Figure 3 shows the pesticide distribution of the nozzle 200 under pressure 1.2 MPa, speed $1.21 \mathrm{~km} / \mathrm{h}$, and spray control distance $960 \mathrm{~mm}$. Figure 4 shows the pesticide distribution of the static nozzle 320 under pressure 1.2 MPa. Figure 5 shows the pesticide distribution of the nozzle 320 under pressure 1.2 $\mathrm{MPa}$, speed $1.21 \mathrm{~km} / \mathrm{h}$, and spray control distance $960 \mathrm{~mm}$.
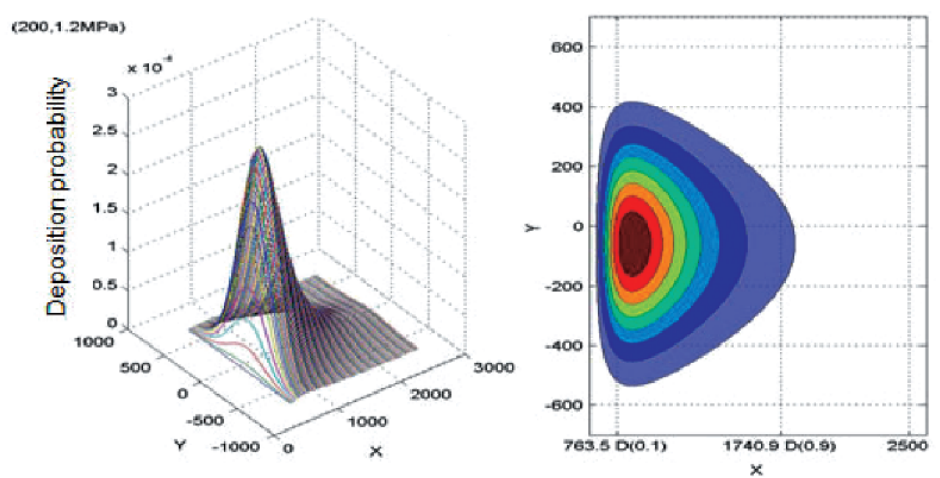

Figure 2. Static nozzle 200 distribution with pressure $1.20 \mathrm{MPa}$

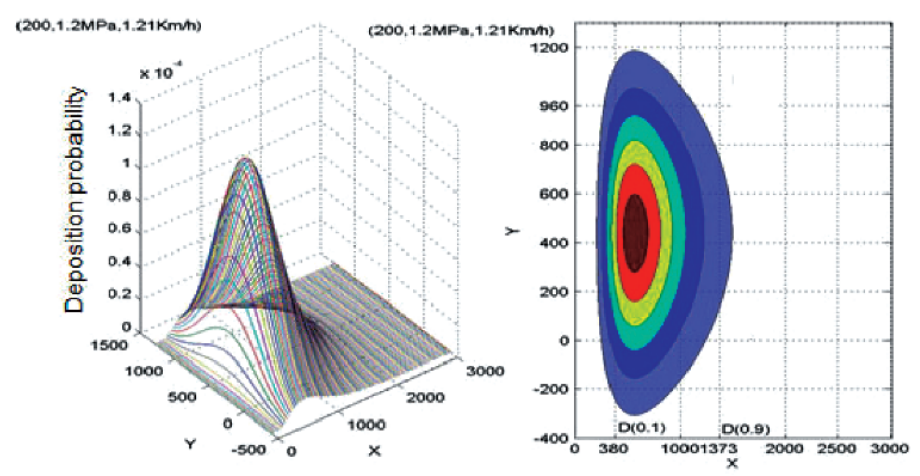

Figure 3. Nozzle 200 distribution with speed $1.21 \mathrm{~km} / \mathrm{h}$ 


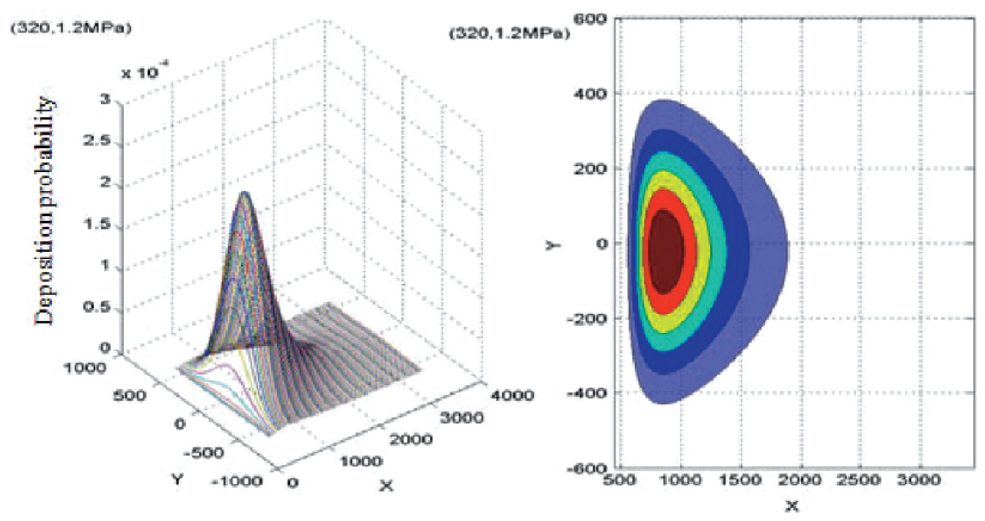

Figure 4. Static nozzle 320 distribution with pressure $1.20 \mathrm{MPa}$

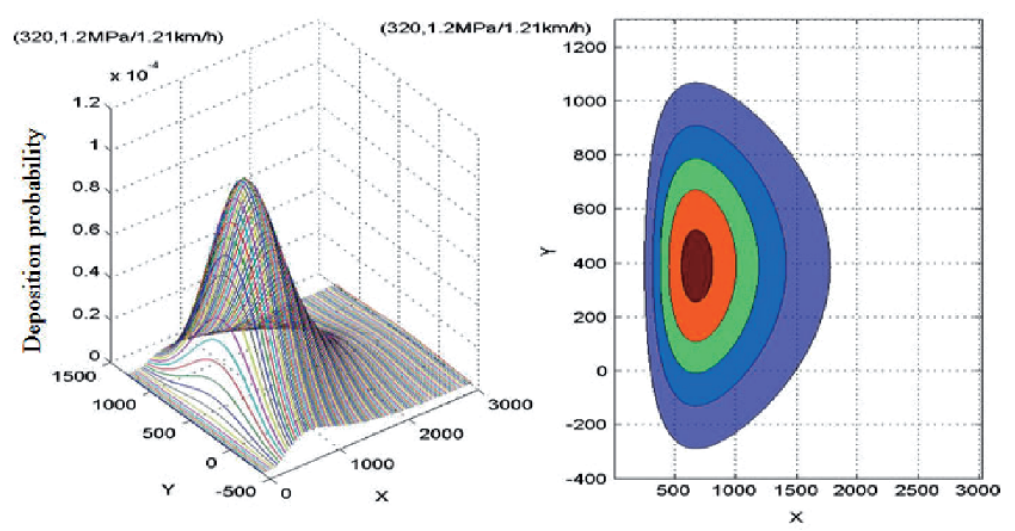

Figure 5. Nozzle 320 distribution with speed $1.21 \mathrm{~km} / \mathrm{h}$

\section{CONCLUSIONS}

Based on the spray experiment datum the pesticide deposition distribution can be simulated on computer by using the probability density function and inverse function of cumulative distribution in Matlab Statistic Toolbox, then the minimum spray distance $D(0.1)$, the maximum distance $D(0.9)$ and the average distance $D(0.5)$ can be computed and the region of the spray solution deposition under different speed and pressure can be calculated.

The simulation result shows that the minimum spray deposition distance $D(0.1)$, the average distance $D(0.5)$ and the maximum distance $D(0.9)$ have 
linearity increased with the increase of the spray pressure, and have linearity decreased with the increase of the spray speed. The influence of pressure and speed on the average distance is smaller than that on the maximum distance, but bigger than that on the minimum distance. The simulation and calculation to the two nozzle spray test shows that, among a certain scope of low speed, with the increase of spray speed, the spray deposition range $D$ (0.9)- $D(0.1)$ is to remain stable. But, when the type 200 nozzle is under the speed above $1.20 \mathrm{~km} / \mathrm{h}$ and the type 300 nozzle is under the speed above $2.22 \mathrm{~km} / \mathrm{h}$, the deposition range decreases significant Figure 6 shows spray distance standard deviation changing with the spray ground speed for nozzle 200 and 320 under spray pressure 1.2MPa respectiveIt is known that the nozzle with big VMD has a wide spray ground speed control range. This simulation result is consistent to the theoretical analysis.
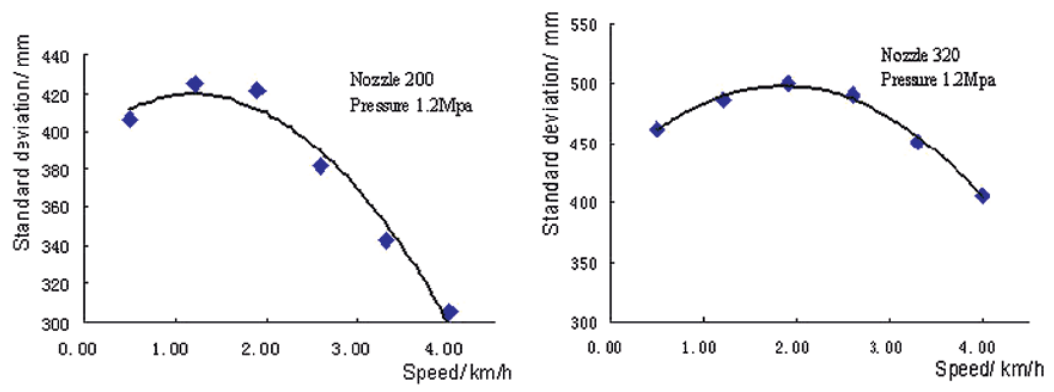

Figure 6. Spray distance standard derviation with spray ground speed

\section{REFERENCES}

China Agricultural University. 1999. Agricultural Machinery (1) [C]. Beijing: China Agriculture Publishing House

Gillis K P, Giles D K, Slaughter D. C, et al. 2003. Distance-Based Control System For Machine Vision-Based Selective praying [J]. Transactions of the ASAE. 45(5): 1255-1262

Liu Dianrui, Liu Gang. 1995. Fitting three parameters on the lognormal distribution at the third parameter C optimization method [J]. Geological exploration. 35 (5): 43-48

Molto E, Martin B, Gutierrez A. 2000. Design and testing of an automatic machine for spraying at a constant distance from the tree canopy $[\mathrm{J}]$. Journal of Agricultural Engineering Research. 77(4): 379-384

Su Jin, Zhang Lian, Liu Bo. 2000. Matlab Toolbox Application [C]. Beijing: Electronics Industry Publishing House

Wang Wanzhang. 2005. Pesticide Distribution Test of the Profile Modeling Spray Based on Ultrasonic Sensor for Fruit Tree [D]. Guangzhou, College of Engineering, South China Agricultural University 